[B3-11]

\title{
STUDY ON A ROBUST CONTROLLER FOR ELECTRO-PNEUMATIC SERVO SYSTEM
}

\author{
Zhou Hong, Lu Yongxiang \\ Institute for Fluid Power Transmission and Control \\ Zhejiang University \\ Hangzhou, China
}

\section{ABSTRACT}

The ready studies show that the characteristics of an electropneumatic servo control system (EPSCS) can be improved remarkably by employing of the state-feedback technique. But the control error of such a system cannot be ignored, especially when there exits large external disturbance, and its stiffness is not satisfied.

$$
\text { In this paper, the authors }
$$
propose a robust control scheme, and develop a design method for robust controller, when the

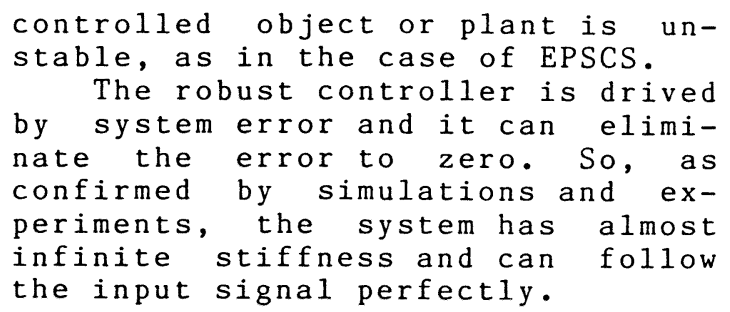

\section{KEYWORDS}

Pneumatics

Robust control

Servo control

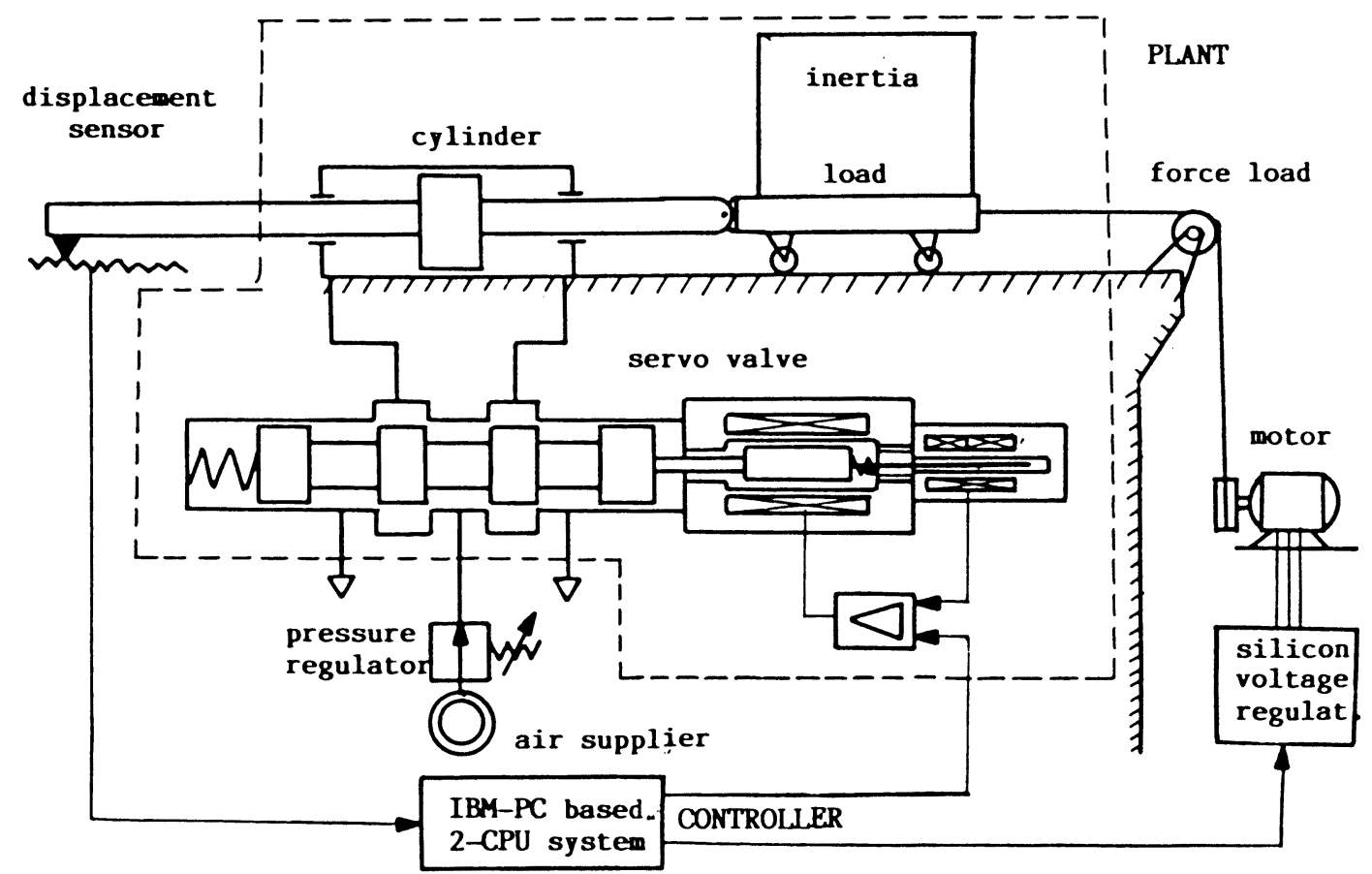

Fig. l Electro-pneumatic servo control system 


\section{INTRODUCTION}

With the development of modern control theory, measurement technique and computer science, electropneumatic servo control systems (EPSCSs) find more and more applications in robots, FMLs and many other fields. A lot of researchers and companies pay attention to such systems and great deal of achievements have got.

Figure 1 shows an EPSCS studied by the authors, it is composed of an electro-pneumatic servo valve, a cylinder, a displacement sensor and a controller. We consider the electro-pneumatic servo valve and the cylinder with inertia load (framed by dotted line in fig.1) as plant of the EPSCS. The mathematical model of the plant can be founded with thoeretical analysis and identification (1), and its linearized transfer function is derived as follows:

$$
G(S)=\frac{K v}{S\left(S^{2} / W^{2}+2 \xi S / W n+1\right)}
$$

Simulations and experiments show that the natural frequency $W n$ and damping radio $\xi$ of the studied system are as low as $21 \mathrm{~Hz}$ and 0.01 respectively. This makes its control very difficult. So, it is necessary to adjust the plant. Researches reveal that the characteristics of plant of an EPSCS can be improved remarkably by a statefeedback controller(2). Figure 2 gives a comparison of performances of the plants before and after adjustment by means of statefeedback.

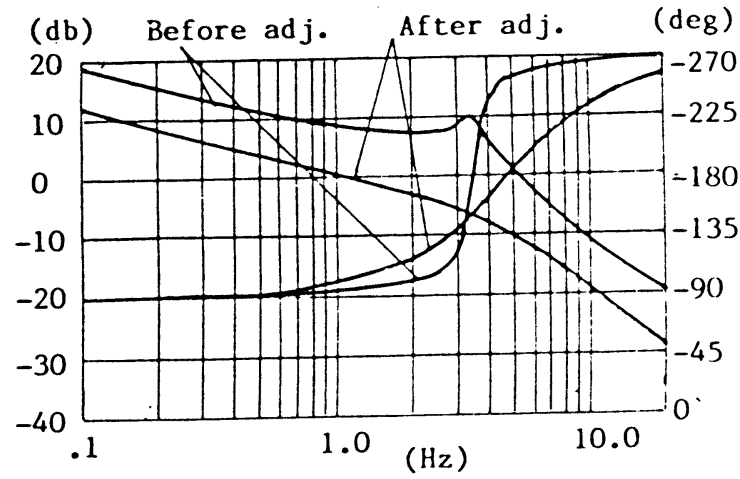

Fig. 2 Performances of the plants before and after adjustment

The robustness of a control system can be evaluated by "return difference". The greater the return difference is, the better the robustness of the system is. The return difference of an EPSCS with state-feedback controller can be defined by the following equation:

$T(w j)=1+K(w j I-A)^{-1} B$

where:

$\mathrm{T}(\mathrm{w} j) \longrightarrow$ return difference

$\mathrm{K}$ Feedback gain victor

It is clear by Eq. (2) that the robustness of an EPSCS with statefeedback controller can be improved by increment of the feedback gain victor $K$. But unfortunately the $K$ cannot be too large, otherwise the system will be unstable due to external disturbances and saturation. So, the robustness of such an EPSCS is limited and the positioning accuracy and stiffness of it can not meet demands of some application field, especially when high accuracy is desired.

In this paper, the authors offer a new control scheme with robust control concept. The authors discuss briefly the principle of the robust controller at first, and then present its design procedure, at last give the experiment results and research conclusions.

PRINCIPLE OF THE ROBUST CONTROLLER TO BE USED (3)

The state and output equations of the plant under consideration are assumed as:

$\dot{\mathrm{X}}=\mathrm{A} \mathrm{X}+\mathrm{B} \mathrm{u}+\mathrm{D} w$

$\mathrm{Y}=\mathrm{CX}$

where the state vector $x \in R^{n}$, the input vector $u \in R^{m}$, the output vector $Y \in R^{1}$, the unmeasurable constant disturbance vector $w \in R$, and all the eigenvalues of the $\mathrm{n} * \mathrm{n}$

plant matrix $A \in R$ lie in the open left half-plane.

Suppose the control-law for the plant is error-actuated and has the form as:

$u=\alpha \cdot \varepsilon K^{\prime} e+\varepsilon K^{\prime} z$

where the error $e=V-Y \in R^{1}$, system input $v \in R$, $z=\int_{0}^{t} e \cdot d t \in R^{1}$,

the scalars $\alpha, \varepsilon \in R$, and the controller gain matrix $K^{\prime} \in \mathrm{R}^{\mathrm{m}^{*} \mathrm{I}}$. It is easy to set up the closed-loop state equation as: 
$\left[\begin{array}{l}\dot{X} \\ \dot{z}\end{array}\right]=\left(\begin{array}{cc}A-\alpha \varepsilon B K^{\prime} C & \varepsilon B K \\ -C & 0\end{array}\right)\left[\begin{array}{l}X \\ z\end{array}\right]+\left(\begin{array}{c}\alpha \varepsilon B K \\ I_{e}\end{array}\right) V+\left.\right|_{0} ^{D}{ }_{0} w$

Therefore, if only provided that $\alpha$, $\varepsilon$ and $K^{\prime}$ are such that all the eigenvalues of the closed-loop plant matrix in equation (4) lie in the open left half-plane, then $\lim \dot{z}=0$ and consequently $\lim e=0$, $t \rightarrow \infty \quad t \rightarrow \infty$

so that set-point tracking occurs simultaneously with disturbance rejection.

The closed-loop characteristic equation can be expressed in the form:

$-1$

$\phi(\lambda)=\left|S(\lambda) \| \lambda I_{\ell}+\varepsilon(\alpha \lambda+1) S^{-1}(\lambda) U(\lambda) K^{\prime}\right|$

where $S(\lambda)$ and $U(\lambda)$ are polynomial matrix such that $G(\lambda)=S(\lambda) \cdot U(\lambda)$ and $\left|S^{\top}(\lambda)\right|=|\lambda \cdot \operatorname{In}-A|$. Hence, express: $K^{\prime}=G^{\top}\left(G G^{\top}\right) \Sigma$

where $\Sigma=\operatorname{diag}\left(\sigma_{1}, \sigma_{2}, \ldots, \sigma_{e}\right), \sigma_{i}>0$ $(i=1,2, \ldots 1)$, it will follow from equations (4) and (5) that $Z c=Z, U Z_{2}$ is the set of closed-loop characteristic roots and

$Z=\{\lambda \in C:|\lambda \cdot \operatorname{In}-A+o(\varepsilon)|=0\}$

$Z=\left\{\lambda \in C: \mid \lambda \cdot I_{l}+\varepsilon \Sigma+o\left(\varepsilon^{2}\right)=0\right\}$

These expressions indicate that both $Z_{1}$ and $Z_{2}$ are in the left open half-plane since $\left|\lambda I_{n}-A\right|=0$ is the characteristic equation of the open-loop asymptotically stable plant and since it is a positive diagonal matrix. The introduction of error-actuated controllers governed by Eq. (4) and (7) accordingly ensure that set-point tracking occurs simultaneously with disturbance rejection when the gain parameter $\varepsilon \in\left(0, \varepsilon^{*}\right]$.
DESIGN PROCEDURE OF ROBUST CONTROLLER FOR EPSCSS

Considering the studied EPSCS had the transfer function as Eq. (1), the plant is unstable due to an integral unit is involved, so we could not follow the design procedure discussed above directly.

Figure 3 illustrates the strategy employed by authors to transform the plant into a stable one. An optimum state-feedback controller, which is designed according to an optimal quadric index with help of a software named $\operatorname{PCSCAD(4)}$, is used. We call the system which composes of the plant and the feedback controller an "extended plant". The state and output equations of the extended plant can be derived as:

$\dot{X}=A^{\prime} X+B^{\prime} u+D^{\prime} w$

$\mathrm{Y}=\mathrm{CX}$

where

$A^{\prime}=\left[\begin{array}{ccc}0 & 1 & 0 \\ 0 & 0 & 1 \\ -29621 & -2868 & -116\end{array}\right]$

The eingevalues of matrix $A^{\prime}$ are:

$\lambda_{1}=-86.93, \quad \lambda_{2,3}=-14.5 \pm 23.2 j$

Al1 of the eingevalues of the extended plant matrix $A^{\prime}$ are in open left half-plane and the extended plant is asymptotically stable. So, to design the robust controller, we can follow the procedure described in the last chapter.

Here, we take the robust control-law in the form of:

$\mathrm{u}=\mathrm{k}^{\prime} \mathrm{z}$

$z=\int_{0}^{t} e d t$

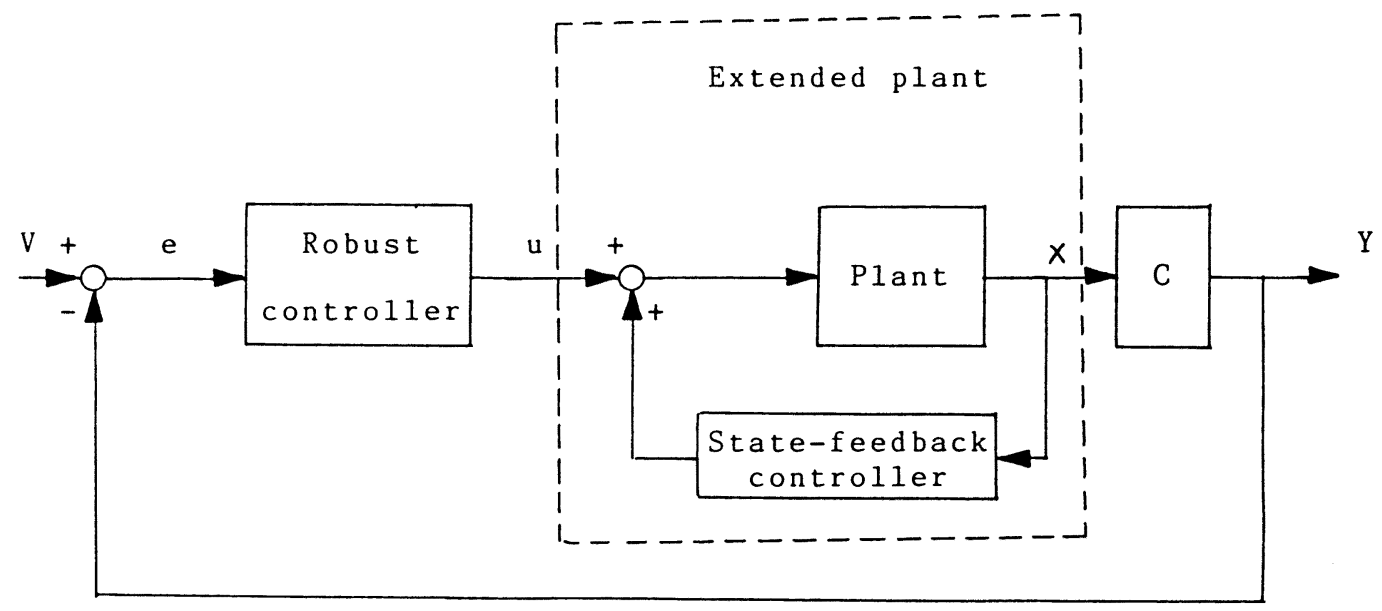

Fig. 3 Strategy of robust controller 
The parameter $k^{\prime}$ is so selected that all the eigenvalues of the closed-loop plant matrix

$$
\left[\begin{array}{cccc}
0 & 1 & 0 & 0 \\
0 & 0 & 1 & 0 \\
-29621 & -2868 & -116 & 164 k^{\prime} \\
-1 & 0 & 0 & 0
\end{array}\right]
$$

are in the open left half-plane. For an example,the eigenvalues of the above matrix are as follows:

$\lambda_{1}=-4.26, \lambda_{2}=-86.75, \lambda_{3,4}=14.2 \pm 23 j$

if $k^{\prime}=500$, they are all in the open left half-plane. Then, as is proved above, set-point tracking occurs with disturbance rejection. Optimum value of $k$ is obtained by a real time optimization test aided by computer(4).

\section{RESULTS AND CONCLUSIONS}

A comparison between robust control and state-feedback control is made for investigating the effect of the robust controller. Figure 4 and 5 are the responses of

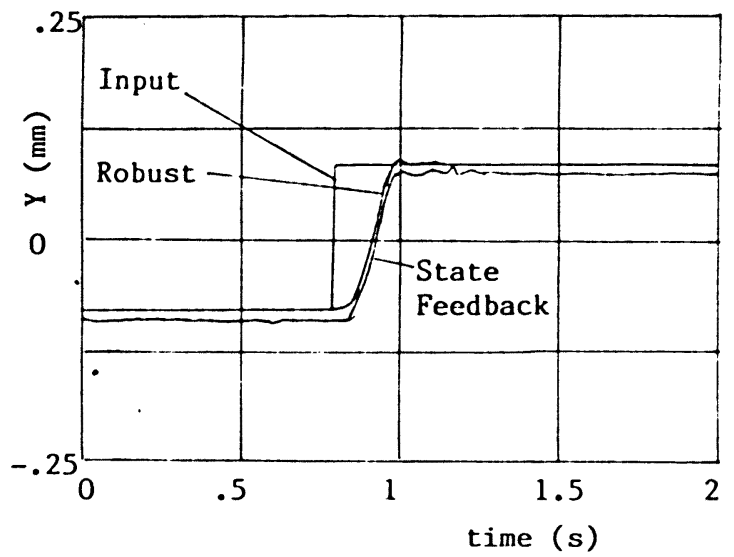

Fig. 4 Comparison of two control methods by step response

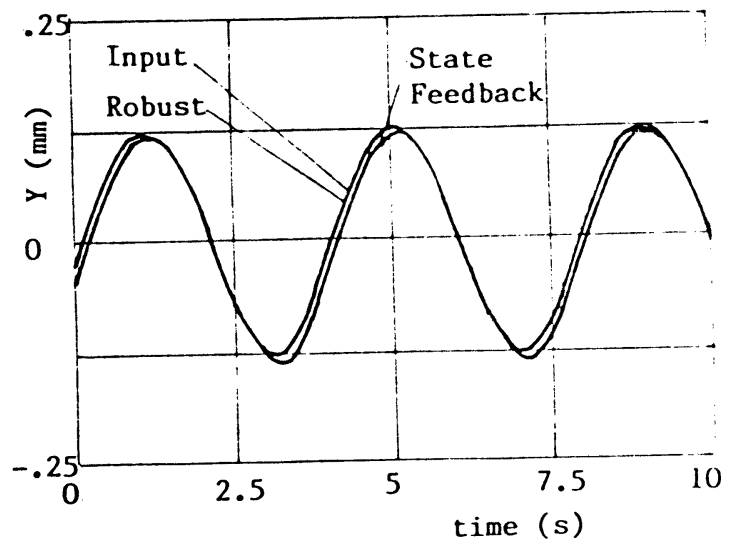

Fig. 5 Comparison of two control methods by sinus response the two systems to a step and a sinus inputs. It is clear by the figure that the system with robust controller has higher tracking accuracy. Further experiments reveal that the tracking accuracy of the EPSCS with robust controller is determined almost only by the accuracy of displacement sensor.

Figure 6 shows the stiffness of the two systems and ensure that the robust controller can raise the stiffness of EPSCS to almost infinite.

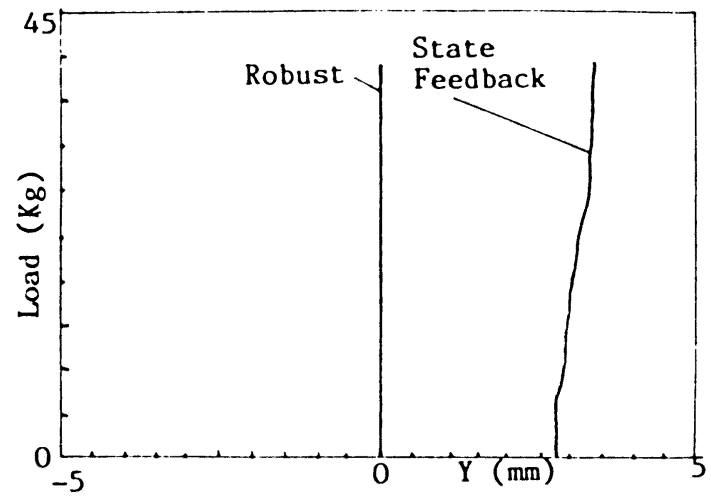

Fig. 6 Comparison of two control methods by stiffness

Through the discuss, several conclusions are obtained:

1). The characteristics of EPSCS can be improved significantly though state-feedback controller, but the limitation in accuracy still exists.

2). Employ of an state-feedback controller can not only transform the the unstable plant of EPSCS into stable one but also make its dynamic characteristics optimum.

3). The robust controller studied here can improve remarkably the dynamic and static performances of EPSCS.

\section{REFERENCES}

(1). Y. X. Lu, Zhou Hong, Identification of Pneumatic Servo System, $8 \mathrm{th}$ IFAC Symposium on Identification and System Parameter Estimation, 1988, Beijing, P.R. CHINA

(2). R. Schwenzer, Statisches und dynamisches Verhalten zustandsgeregelter Pneumatikantriebe, $0+P$, $1984 \mathrm{Nr} .3$

(3). C.J. Harris and S.A. Billings, Self-tuning and Adaptive Control: Theory and Application, Peter Peregrinus Ltd., Stevenage, UK, and New York, 1981

(4). Zhou Hong, Study on Electropneumatic Servo Control System, Doctoral Thesis, Zhejiang University, 1988 